\title{
Supersymmetry: Boundary conditions and edge states
}

\author{
Nirmalendu Acharyya, ${ }^{1, *}$ Manuel Asorey, ${ }^{2, \dagger}$ A. P. Balachandran, ${ }^{3,4, \$}$ and Sachindeo Vaidya ${ }^{1, \S}$ \\ ${ }^{1}$ CHEP, Indian Institute of Science, Bangalore, Karnataka 560012, India \\ ${ }^{2}$ Departamento de Física Teórica Facultad de Ciencias Universidad de Zaragoza, 50009 Zaragoza, Spain \\ ${ }^{3}$ Department of Physics, Syracuse University, Syracuse, New York 13244-1130, USA \\ ${ }^{4}$ Departamento de Fisica, Universidad de los Andes, Bogotá 4976, Colombia
}

(Received 25 May 2015; published 10 November 2015)

\begin{abstract}
When spatial boundaries are inserted, supersymmetry (SUSY) can be broken. We have shown that in an $\mathcal{N}=2$ supersymmetric theory, all local boundary conditions allowed by self-adjointness of the Hamiltonian break $\mathcal{N}=2$ SUSY, while only a few of these boundary conditions preserve $\mathcal{N}=1$ SUSY. We have also shown that for a subset of the boundary conditions compatible with $\mathcal{N}=1$ SUSY, there exist fermionic ground states which are localized near the boundary. We also show that only very few nonlocal boundary conditions like periodic boundary conditions preserve full $\mathcal{N}=2$ supersymmetry, but none of them exhibits edge states.
\end{abstract}

DOI: $10.1103 /$ PhysRevD.92.105016

PACS numbers: 11.30.Pb, 74.25.-q

\section{INTRODUCTION}

Although supersymmetry (SUSY) as a fundamental theory has eluded experimental evidence to date, there has been a recent revival of interest in the subject because it emerges naturally as an effective theory describing the quantum phase transition at the boundary of topological superconductors [1].

All real physical systems available for experiments are of finite size and with spatial boundaries, which in general reduce the symmetries of the system. Hence it is eminently reasonable to ask if the SUSY of a $(d+1)$-dimensional finite size system (like the topological superconductor) can be obtained by the consistent truncation of a parent SUSY system in full $(d+1)$-dimensional Minkowski spacetime. Although one might expect that insertion of spatial boundaries generically breaks SUSY, we will show that there are certain boundary conditions which do preserve supersymmetry partially. Discussions of boundary conditions in this context assume significance, and a clear classification of such boundary conditions is required. The presence of boundaries, on the other hand, naturally leads to the question of edge states, which, if extant, play a vital role in the physics at the boundary [2].

Boundary conditions in supersymmetric theories have been studied in detail (for example see $[3,4]$ and references therein). We consider this problem from a different perspective. We show that self-adjoint domains of the Hamiltonian are enough to obtain the boundary conditions which preserve (or break) supersymmetry. Further, our main objective is to show the existence of edge states in a supersymmetric theory, which will be relevant in the

\footnotetext{
nirmalendu@cts.iisc.ernet.in †asorey@unizar.es

balachandran38@gmail.com

§vaidya@cts.iisc.ernet.in
}

physics of the newly discovered supersymmetric phase in topological superconductors. For this purpose, a simplified treatment of a noninteracting scalar-fermion model is sufficient. As discussed in [5-8], for the above-mentioned model, it is not difficult to see that a supersymmetric variation in the bulk gives boundary terms which vanish only when Dirichlet or Neumann boundary conditions are chosen for the scalar. It is well known that with Dirichlet or Neumann boundary conditions there are no scalar edge localized states [9]. Nevertheless, we show that there can still be fermionic edge states which do not break supersymmetry.

In [1] it was shown that in the phase that breaks SUSY spontaneously, there are edge states on the surface (i.e., the boundary) of the superconductor. However, it is not obvious whether such edge states exist without breaking SUSY. We will investigate the existence of such edge states when the boundary conditions can be chosen to preserve (some) supersymmetry. As we will show, such "SUSY preserving" edge states do exist, and the ground states in such theories are particularly interesting.

Our focus in this article will be on the insertion of a spatial boundary $\partial M$ in $(d+1)$-dimensional Minkowski space, in such a manner that the resulting space continues to be a $(d+1)$-dimensional manifold $M$ with a boundary $\partial M$ (which can be curved in general). The boundary conditions on the (scalar and spinor) fields on $M$ cannot be chosen arbitrarily. They are obtained by demanding that the scalar and Dirac Hamiltonians ( $H_{s}$ and $H_{D}$ respectively) be selfadjoint. Of these boundary conditions, we expect that only a subset will preserve supersymmetry (at least partially), while generic boundary conditions will break supersymmetry completely.

For $H_{s}$ to be self-adjoint, it is necessary that the scalar Laplacian $\left(-\nabla^{2}+m^{2}\right)$ be self-adjoint [9]. Then, if we demand locality of boundary conditions, the domain $\mathcal{D}_{H_{s}}=$ $\mathcal{D}_{H_{s}^{*}}$ of $H_{s}$ contains all $\Phi \in L^{2}(M)$ satisfying 


$$
\begin{aligned}
{\left[\Phi(x)+i \partial_{n} \Phi(x)\right] } & =U_{B}(x)\left[\Phi(x)-i \partial_{n} \Phi(x)\right], \\
U_{B}^{\dagger}(x) U_{B}(x) & =\llbracket, \quad x \in \partial M,
\end{aligned}
$$

where $\hat{n}$ is the outward normal, $\partial_{n} \equiv \hat{n} \cdot \vec{\nabla}$ is the normal derivative at $\partial M$, and $U_{B}(x)$ is a unitary operator on $\Phi(x)$ [if $\Phi(x)$ is the $N$ component, $U_{B}(x) \in U(N)$ ].

For the choices $U_{B}(x)=-\rrbracket_{N \times N}$ and $U_{B}(x)=\mathbb{\square}_{N \times N}$, we get the Dirichlet and Neumann boundary conditions, respectively. Other choices for $U_{B}(x)$ give more general boundary conditions,

$$
\begin{aligned}
{\left[\partial_{n} \Phi-\kappa \Phi\right](x) } & =0, \quad \kappa=i\left(\llbracket+U_{B}\right)^{-1}\left(\llbracket-U_{B}\right), \\
\kappa(x)^{\dagger} & =\kappa(x),
\end{aligned}
$$

whenever $U_{B}$ does not have unit eigenvalues.

To discuss the self-adjointness of $H_{F} \equiv i \gamma^{0} \gamma^{j} \partial_{j}-m \gamma^{0}$, we start by defining two chiralities (on the boundary) for the Dirac spinors $\Psi$ :

$$
\Psi_{ \pm} \equiv \frac{1}{2}\left(1 \pm \gamma^{0} \vec{\gamma} \cdot \hat{n}\right) \Psi
$$

The $\gamma$-matrices here obey

$$
\left\{\gamma^{\mu}, \gamma^{\nu}\right\}=2 \eta^{\mu \nu}, \quad \gamma^{0 \dagger}=\gamma^{0}, \quad \gamma^{j \dagger}=-\gamma^{j},
$$

where $\mu, \nu=0,1 \cdots d, \quad j=1,2 \cdots d, \quad$ and $\quad \eta^{\mu \nu}=$ $\operatorname{diag}(1,-1,-1, \cdots-1)$.

The essential self-adjointness of $H_{F}$ requires that the domains $\mathcal{D}_{H_{F}}$ and $\mathcal{D}_{H_{F}^{*}}$ coincide. If $M$ is compact, the most general self-adjoint extension fulfilling the local boundary condition is given by $\Psi \in W^{1,2}(M) \otimes \mathbb{C}^{N}$ satisfying $[9,10]$

$$
\begin{aligned}
\left(\Psi_{+}-U_{F} \gamma^{0} \Psi_{-}\right)_{\partial M} & =0, \quad U_{F}^{\dagger} U_{F}=1, \\
{\left[U_{F}, \gamma^{0} \vec{\gamma} \cdot \hat{n}\right] } & =0 .
\end{aligned}
$$

In Sec. VI, we consider also nonlocal boundary conditions. They include in particular periodic and antiperiodic boundary conditions on bosons and fermions. We also briefly consider the possibility that the boundary conditions are partly local and partly nonlocal.

We now analyze these general observations in various dimensions.

\section{II. $(1+1)$ DIMENSIONS}

In the full $(1+1)$-dimensional Minkowski spacetime, the simplest theory of a complex scalar $\Phi$ (with the number of components $N=1$ ) and a Dirac fermion $\Psi$ is $\mathcal{N}=2$ supersymmetric [11]. It is described by the action

$$
S=\int_{-\infty}^{\infty} d x_{0} \int_{-\infty}^{\infty} d x_{1}\left(\mathcal{L}_{S}+\mathcal{L}_{D}\right),
$$

where

$$
\begin{gathered}
\mathcal{L}_{S}=\frac{1}{2}\left(\partial^{\mu} \Phi^{*} \partial_{\mu} \Phi-m^{2} \Phi^{*} \Phi\right), \\
\mathcal{L}_{F}=\frac{1}{2}\left(i \bar{\Psi} \gamma^{\mu} \partial_{\mu} \Psi-m \bar{\Psi} \Psi\right) .
\end{gathered}
$$

The SUSY transformations are

$$
\begin{aligned}
& \delta \Phi=\bar{\epsilon} \Psi, \quad \delta \Psi=-i \gamma^{\mu} \epsilon \partial_{\mu} \Phi-m \epsilon \Phi, \\
& \delta \Phi^{*}=\bar{\Psi} \epsilon, \quad \delta \bar{\Psi}=i \bar{\epsilon} \gamma^{\mu} \partial_{\mu} \Phi^{*}-m \bar{\epsilon} \Phi^{*},
\end{aligned}
$$

with

$$
\epsilon=\left(\begin{array}{l}
\epsilon_{1} \\
\epsilon_{2}
\end{array}\right)
$$

where $\epsilon_{i}$ 's are Grassmann constants and $\bar{\epsilon}=\epsilon^{\dagger} \gamma^{0}$.

We consider the same system in a $(1+1)$-dimensional manifold $M$ with spatial boundary $\partial M$ :

$$
M=\left\{\left(x^{0}, x^{1}\right): x^{1} \leq 0\right\} .
$$

The action is given by

$$
S=\int_{-\infty}^{\infty} d x_{0} \int_{-\infty}^{0} d x_{1}\left(\mathcal{L}_{S}+\mathcal{L}_{D}\right)+S_{B}
$$

and $S_{B}$ are the boundary terms on $\partial M$ as in $[5,6]$ :

$S_{B}=\frac{1}{4} \int_{-\infty}^{\infty} d x_{0}\left(\Phi^{*} \partial_{n} \Phi+\left(\partial_{n} \Phi^{*}\right) \Phi-i \bar{\Psi} \vec{\gamma} \cdot \hat{n} \Psi\right)_{\partial M}$.

The boundary terms are analogous to the GibbonsHawking term. Its goal is to give rise to local equations of motion independently of boundary conditions on the fields.

The boundary conditions (1) and (5) are imposed on $\Phi$ and $\Psi$ at the boundary points $x^{1}=0$. Out of this family of allowed boundary conditions, which ones are consistent with the SUSY transformations (9)?

The SUSY transformation $\delta \Phi$ and $\delta \Psi$ must obey (1) and (5) on the boundary. The variation of the scalar field on the boundary leads to

$$
\left[\left(1-U_{B}\right) \bar{\epsilon} \Psi+i\left(1+U_{B}\right) \bar{\epsilon}\left(\partial_{1} \Psi\right)\right]_{\partial M}=0,
$$

$U_{B}$ in this case being a phase. The variation of $\Psi$ on the boundary yields

$$
\begin{aligned}
& {\left[-i\left(\gamma^{\mu} \epsilon\right)_{+} \partial_{\mu} \Phi-m \epsilon_{+} \Phi\right]_{\partial M}} \\
& \quad=\left[-i U_{F} \gamma^{0}\left(\gamma^{\mu} \epsilon\right)_{-} \partial_{\mu} \Phi-m U_{F} \gamma^{0} \epsilon_{-} \Phi\right]_{\partial M},
\end{aligned}
$$

which leads to 


$$
\begin{aligned}
& {\left[i \gamma^{\mu} \epsilon_{-} \partial_{\mu} \Phi+m \epsilon_{+} \Phi\right]_{\partial M}} \\
& \quad=\left[i \gamma^{0} \gamma^{\mu} U_{F} \epsilon_{+} \partial_{\mu} \Phi+m U_{F} \gamma^{0} \epsilon_{-} \Phi\right]_{\partial M} .
\end{aligned}
$$

It can be easily checked that (13) and (15) are incompatible if $U_{B} \neq \pm 1$ (Dirichlet or Neumann). Therefore if Robin boundary conditions are imposed on scalars $[\kappa \neq 0$ or $\kappa \neq \infty$, where $\kappa$ is defined in (2)] in the $(1+1)$ dimensional theory, then $\mathcal{N}=2$ SUSY is completely broken.

Dirichlet and Neumann boundary conditions: However, Dirichlet and Neumann boundary conditions on the scalar are consistent with SUSY. To show that let us consider the massless and massive cases separately.

Massless case: If we impose the Dirichlet $\left(U_{B}=-1\right)$ or Neumann $\left(U_{B}=1\right)$ condition on the massless scalar $\Phi$, the supersymmetry condition (15) leads to

Dirichlet: $\left.i \gamma^{1} \epsilon_{-} \partial_{1} \Phi\right|_{\partial M}=-\left.i \gamma^{0} \gamma^{1} U_{F} \epsilon_{+} \partial_{1} \Phi\right|_{\partial M}$

Neumann: $\left.i \gamma^{0} \epsilon_{-} \partial_{0} \Phi\right|_{\partial M}=-\left.i \gamma^{0} \gamma^{0} U_{F} \epsilon_{+} \partial_{0} \Phi\right|_{\partial M}$,

which yields the following condition on the SUSY parameter $\epsilon$ :

$$
\begin{aligned}
& \text { Dirichlet: } \epsilon_{-}=-\gamma^{0} U_{F} \epsilon_{+}, \\
& \text {Neumann: } \epsilon_{-}=\gamma^{0} U_{F} \epsilon_{+} .
\end{aligned}
$$

On the other hand, these choices $U_{B}= \pm 1$ in (13) give

$$
\text { Dirichlet: }\left.\bar{\epsilon} \Psi\right|_{\partial M}=0 \text {, }
$$

$$
\text { Neumann: }\left.\bar{\epsilon} \partial_{1} \Psi\right|_{\partial M}=0 \text {. }
$$

For the Dirichlet boundary condition on the scalar, the condition (19) is trivially satisfied when the boundary condition (1) and the condition (17) on $\epsilon$ are used.

For Neumann boundary condition on the scalar, the condition (20) [along with (18)] yields a new boundary condition

$$
\left.\left(\partial_{1} \Psi\right)_{+}\right|_{\partial M}=-\left.U_{F} \gamma^{0}\left(\partial_{1} \Psi\right)_{-}\right|_{\partial M} .
$$

However, the appearance of this extra boundary condition is not surprising in a supersymmetric theory. The supercharge $Q$ obeys

$$
\{Q, \bar{Q}\} \Psi \propto H_{F} \Psi .
$$

Hence, it is necessary to ensure that $\left(H_{F} \Psi\right)$ is also in the domain of $H_{F}$. Otherwise SUSY will change the domain of $H_{F}$. Hence, we must also impose

$$
\left.\left(H_{F} \Psi\right)_{+}\right|_{\partial M}=\left.U_{F} \gamma^{0}\left(H_{F} \Psi\right)_{-}\right|_{\partial M}
$$

which in the massless $(1+1)$-dimensional case reduces to $(21)$.

Therefore, the Dirichlet (or Neumann) boundary condition on massless $\Phi$ is consistent with the supersymmetry transformations and the system is supersymmetric. But owing to the relation (17) [or (18)], the system only has $\mathcal{N}=1$ supersymmetry.

Massive case: If the Dirichlet boundary condition $\left(U_{B}=-1\right)$ is imposed on the scalar, the supersymmetry condition (13) and the boundary condition (5) lead to

$$
\epsilon_{-}=-\gamma^{0} U_{F} \epsilon_{+} .
$$

With the Dirichlet boundary condition on $\Phi$ and (24), it is easy to see from (15) that

$$
\delta \Psi_{+}=U_{F} \gamma^{0} \delta \Psi_{+}
$$

is satisfied. Therefore, this choice of boundary conditions is consistent with SUSY.

If the Neumann boundary condition $\left(U_{B}=1\right)$ is imposed on the scalar, the supersymmetry condition (13) gives

$$
\left.\bar{\epsilon}\left(\partial_{1} \Psi\right)\right|_{\partial_{M}}=0,
$$

while (15) leads to

$$
\begin{aligned}
& {\left[i \gamma^{0} \epsilon_{-} \partial_{0} \Phi+m \epsilon_{+} \Phi\right]_{\partial M}} \\
& \quad=\left[i \gamma^{0} \gamma^{0} U_{F} \epsilon_{-} \partial_{0} \Phi+m U_{F} \gamma^{0} \epsilon_{-} \Phi\right]_{\partial M} .
\end{aligned}
$$

In contrast to the massless case, here, because of the extra mass term in (27), this cannot be made compatible with (26) just by a condition on $\epsilon$. However, the two can be made compatible by imposing the further condition $U_{F}=U_{F}^{\dagger}$. Hence the Neumann boundary condition on the massive scalar in $(1+1)$ dimensions is consistent with SUSY when

$$
\epsilon_{-}=\gamma^{0} U_{F} \epsilon_{+}, \quad U_{F}=U_{F}^{\dagger} .
$$

As a result, in the massive $(1+1)$-dimensional theory, imposing the Dirichlet or Neumann boundary condition on the scalar breaks $\mathcal{N}=2$ SUSY to $\mathcal{N}=1$ SUSY.

With the choice $\gamma^{0}=\sigma_{2}$ and $\gamma^{1}=i \sigma_{1}$, in the $(1+1)$ dimensional massless case and in the massive case with Dirichlet boundary condition, the most general $U_{F}$ satisfying (5) is

$$
U_{F}=\left(\begin{array}{cc}
e^{i \theta} & 0 \\
0 & e^{i \tilde{\theta}}
\end{array}\right), \quad \theta, \tilde{\theta} \in \mathbb{R}
$$

For the massive case with the Neumann boundary condition, because of the condition (28), only $\theta=\tilde{\theta}=0$ or $\pi$ are allowed by supersymmetry and hence the only $U_{F}$ 's that preserve SUSY partially are 


$$
U_{F}= \pm\left(\begin{array}{ll}
1 & 0 \\
0 & 1
\end{array}\right)
$$

In the case of a system with two boundaries, like an open superstring, the standard boundary conditions are Neumann boundary conditions for scalars and (30) for fermions at both boundaries. The case with $U_{F}=I$ at both boundaries is called the Ramond (R) sector, while the case with $U_{F}=I$ at one boundary and $U_{F}=-I$ at the other is termed the Neveu-Schwarz (N-S) sector.

Using the above in (17), (18), (24), and (28), we get

$$
\begin{aligned}
& \text { Dirichlet: } \epsilon_{1}=-i e^{-i \theta} \epsilon_{2}, \\
& \text { Neumann (massless): } \epsilon_{1}=i e^{-i \theta} \epsilon_{2}, \\
& \text { Neumann (massive) }: \epsilon_{1}= \pm i \epsilon_{2} .
\end{aligned}
$$

The closure of the SUSY algebra is given by

$$
\left[\delta_{\epsilon}, \delta_{\eta}\right]=-i\left(\epsilon^{\dagger} \eta-\eta^{\dagger} \epsilon\right) \partial_{0}+2 m(\bar{\epsilon} \eta-\bar{\eta} \epsilon) .
$$

The unbroken $\mathcal{N}=2$ SUSY algebra in $(1+1)$ dimensions is generated by two supercharges $Q_{ \pm}$:

$$
\begin{aligned}
& \left\{Q_{ \pm}, \bar{Q}_{ \pm}\right\}=\mathcal{P}_{0} \pm \mathcal{P}_{1}, \quad\left\{Q_{-}, \bar{Q}_{+}\right\}=\mathcal{Z}, \\
& \left\{Q_{+}, \bar{Q}_{-}\right\}=\overline{\mathcal{Z}}, \quad\left[P_{\mu}, \mathcal{Z}\right]=0,
\end{aligned}
$$

where $\mathcal{Z}$ is the central charge. In the $\mathcal{N}=1$ theory, as the SUSY parameter satisfies (17) or (18) in the massless case and (24) or (28) in the massive case, the super charges are

$$
\begin{aligned}
& \text { Dirichlet: } Q=Q_{+}+i e^{i \theta} Q_{-}, \\
& \text {Neumann (massless) }: Q=Q_{+}-i e^{i \theta} Q_{-}, \\
& \text {Neumann (massive) }: Q=Q_{+} \pm i Q_{-},
\end{aligned}
$$

satisfying

Dirichlet: $\{Q, \bar{Q}\}=2 \mathcal{P}_{0}-i\left(e^{-i \theta} \overline{\mathcal{Z}}-e^{i \theta} \mathcal{Z}\right)$,

Neumann (massless): $\{Q, \bar{Q}\}=2 \mathcal{P}_{0}+i\left(e^{-i \theta} \overline{\mathcal{Z}}-e^{i \theta} \mathcal{Z}\right)$,

Neumann (massive): $\{Q, \bar{Q}\}=2 \mathcal{P}_{0} \pm i(\overline{\mathcal{Z}}-\mathcal{Z})$,

and

$$
\left[Q, \mathcal{P}_{0}\right]=0 \text {. }
$$

In (34), the mass term is the central charge contribution (i.e., the massless theory has $\mathcal{Z}=0$ ). In the massless case, this term vanishes and we get the usual $\mathcal{N}=1$ SUSY algebra. But in the massive case, the central charge term can be absorbed by rescaling $\mathcal{P}_{0}$ and the usual $\mathcal{N}=1$ SUSY algebra can be recovered:
Dirichlet (massive) $: \tilde{\mathcal{P}}_{0}=\mathcal{P}_{0}-\frac{i}{2}\left(e^{-i \theta} \overline{\mathcal{Z}}-e^{i \theta} \mathcal{Z}\right)$,

Neumann (massive): $\tilde{\mathcal{P}}_{0}=\mathcal{P}_{0} \pm \frac{i}{2}(\overline{\mathcal{Z}}-\mathcal{Z})$,

$$
\{Q, \bar{Q}\}=2 \tilde{\mathcal{P}}_{0}, \quad\left[Q, \tilde{\mathcal{P}}_{0}\right]=0 .
$$

Hence, if the theory is massless, the $\mathcal{N}=2$ SUSY is broken to a family (characterized by $\theta$ and $\tilde{\theta}$ ) of $\mathcal{N}=1$ supersymmetric theories by introducing the boundary with a Dirichlet or Neumann boundary condition on the scalar.

On the other hand, if the theory is massive, in the presence of a boundary with only the Dirichlet boundary condition on the scalar, it breaks $\mathcal{N}=2$ SUSY to a family of $\mathcal{N}=1$ supersymmetric theories. In the case of Neumann boundary conditions, $\mathcal{N}=2$ SUSY is broken to one of the two possible $\mathcal{N}=1$ SUSY theories, depending on the fermionic boundary conditions (i.e., only when $U_{F}= \pm 1$, and for any other choice of $U_{F}$, SUSY is completely broken). Any other boundary condition on the scalar breaks SUSY completely.

\section{A. Variation of the action}

One can verify that the above results can be simply rederived by requiring invariance of the full action. Indeed, the variation of the action (11) under SUSY yields

$$
\begin{aligned}
\delta S= & \frac{1}{4} \int_{\partial M} d x^{0}\left[\Phi^{*}\left(\bar{\epsilon} \partial_{1} \Psi\right)+\left(\partial_{1} \bar{\Psi} \epsilon\right) \Phi\right. \\
& \left.-\bar{\Psi} \gamma^{1} \gamma^{0} \epsilon \partial_{0} \Phi+\bar{\epsilon} \gamma^{1} \gamma^{0} \Psi \partial_{0} \Phi^{*}\right]_{x_{1}=0} \\
& +\frac{i m}{4} \int_{\partial M} d x^{0}\left[\bar{\Psi} \gamma^{1} \epsilon \Phi-\bar{\epsilon} \gamma^{1} \Psi \Phi^{*}\right]_{x_{1}=0}
\end{aligned}
$$

which does not vanish with an arbitrary choice of boundary condition. However, it can be easily shown that the above vanishes for those boundary conditions which preserve $\mathcal{N}=1$ SUSY (discussed in the previous section).

When the Dirichlet boundary condition is imposed on the scalar,

$$
\left.\Phi\right|_{x^{1}=0}=0,\left.\quad \partial_{0} \Phi\right|_{x^{1}=0}=0,
$$

it is easy to see that $\delta S$ vanishes.

When the Neumann boundary is imposed on the scalar and the theory is massless, the SUSY conditions (18) and (20) give

$$
\left.\bar{\epsilon} \partial_{1} \Psi\right|_{x^{1}=0}=0, \quad \epsilon_{-}=\gamma^{0} U_{F} \epsilon_{+},
$$

and the boundary condition (5) yields 


$$
\begin{aligned}
\left.\bar{\epsilon} \gamma^{1} \gamma^{0} \Psi\right|_{x^{1}=0} & =-\left[\epsilon_{+}^{\dagger} \gamma^{1} \Psi_{-}+\epsilon_{-}^{\dagger} \gamma^{1} \Psi_{+}\right]_{x^{1}=0} \\
& =-\left[\epsilon_{+}^{\dagger} \gamma^{1} \Psi_{-}+\epsilon_{+}^{\dagger} U_{F}^{\dagger} \gamma^{0} \gamma^{1} U_{F} \gamma^{0} \Psi_{+}\right]_{x^{1}=0} \\
& =-\left[\epsilon_{+}^{\dagger} \gamma^{1} \Psi_{-}+\epsilon_{+}^{\dagger} \gamma^{0} \gamma^{1} U_{F}^{\dagger} U_{F} \gamma^{0} \Psi_{+}\right]_{x^{1}=0} \\
& =-\left[\epsilon_{+}^{\dagger} \gamma^{1} \Psi_{-}+\epsilon_{+}^{\dagger} \gamma^{0} \gamma^{1} \gamma^{0} \Psi_{+}\right]_{x^{1}=0} \\
& =-\left[\epsilon_{+}^{\dagger} \gamma^{1} \Psi_{-}-\epsilon_{+}^{\dagger} \gamma^{1} \Psi_{+}\right]_{x^{1}=0} \\
& =0 .
\end{aligned}
$$

Using (44) and (45) in (42), it is easy to check that in the massless case $\delta S$ vanishes.

In the massive theory, when the Neumann boundary condition is imposed on the scalar, along with (44) and (45), $U_{F}$ also satisfies $U_{F}^{\dagger}=U_{F}$. Owing to the last condition on $U_{F}$, it follows that

$$
\begin{aligned}
\left.\bar{\epsilon} \gamma^{1} \Psi\right|_{x^{1}=0} & =\left[\epsilon_{+}^{\dagger} \gamma^{0} \gamma^{1} \Psi_{+}+\epsilon_{-}^{\dagger} \gamma^{0} \gamma^{1} \Psi_{-}\right]_{x^{1}=0} \\
& =\left[\epsilon_{+}^{\dagger} \gamma^{0} \gamma^{1} U_{F} \gamma^{0} \Psi_{-}+\epsilon_{+}^{\dagger} U_{F}^{\dagger} \gamma^{0} \gamma^{0} \gamma^{1} \Psi_{-}\right]_{x^{1}=0} \\
& =\left[\epsilon_{+}^{\dagger} U_{F} \gamma^{0} \gamma^{1} \gamma^{0} \Psi_{-}+\epsilon_{+}^{\dagger} U_{F}^{\dagger} \gamma^{1} \Psi_{-}\right]_{x^{1}=0} \\
& =\left[-\epsilon_{+}^{\dagger} U_{F} \gamma^{1} \Psi_{-}+\epsilon_{+}^{\dagger} U_{F}^{\dagger} \gamma^{1} \Psi_{-}\right]_{x^{1}=0} \\
& =0 .
\end{aligned}
$$

When (44)-(46) are substituted in (42), in the massive case also, we find,

$$
\delta S=0 .
$$

Therefore, these results are consistent with the findings of the previous section.

\section{EDGE STATES IN $(1+1)$ DIMENSIONS}

In these massive $\mathcal{N}=1$ theories, for the choice of $\theta=(2 n+1) \frac{\pi}{2}$ in $(29)$, there are zero-energy fermionic modes:

$\Psi_{e}=G e^{b x_{1}}\left(\begin{array}{c}1 \\ -(-1)^{n}\end{array}\right), \quad b=(-1)^{n} m, \quad n \in \mathbb{Z}$.

$G$ is the normalization constant. These modes are normalizable only for $m>0$ and $n=$ even or $m<0$ and $n=$ odd. If $|m|$ is sufficiently large, the zero modes are exponentially damped in the bulk $x^{1}<0$ and are therefore localized near the boundary. For the scalar $\Phi$, however, there is no zero-energy mode with a Dirichlet boundary condition. Thus the fermionic edge states, when present, are not paired with bosonic edge states. But such unpaired states do not break SUSY as they are zero-energy modes and singlets under SUSY. Consequently, when the boundary conditions are suitably chosen such that the edge states exist, the residual $\mathcal{N}=1$ supersymmetric theory has a fermionic ground state.

It is interesting to note that edge states do exist in Ramond and Neveu-Schwarz sectors in the massive case.
In the massless theory, such fermionic edge states do not exist because there is no mass gap.

\section{IV. $(3+1)$-DIMENSIONS}

In the full $(3+1)$-dimensional Minkowski spacetime, a theory with two complex scalars $\Phi_{a}$ 's $(a=1,2)$ and a Dirac spinor $\Psi$ with the action

$$
\begin{gathered}
S=\int_{-\infty}^{\infty} d^{4} x\left(\mathcal{L}_{\text {kin }}+\mathcal{L}_{m}\right) \\
\mathcal{L}_{\text {kin }}=\frac{1}{2} \partial^{\mu} \Phi_{a}^{\dagger} \partial_{\mu} \Phi_{a}+i \bar{\Psi} \gamma^{\mu} \partial_{\mu} \Psi+\frac{1}{2} F_{a}^{\dagger} F^{a}, \\
\mathcal{L}_{m}=m\left(\frac{i}{2} \Phi_{a}^{\dagger} F^{a}-\frac{i}{2} F_{a}^{\dagger} \Phi^{a}+\bar{\Psi} \Psi\right)
\end{gathered}
$$

is $\mathcal{N}=2$ supersymmetric with a central charge $Z=P^{\mu} P_{\mu}$. Here $F_{a}$ 's are two complex scalar auxiliary fields which are necessary to close SUSY off-shell. The nonzero central charge ensures that particles with spin $>\frac{1}{2}$ are absent from the multiplet (for details see pages 150-152 in [12]). The supersymmetry transformations are

$$
\begin{gathered}
\delta \Phi_{a}=2 \bar{\epsilon}^{a} \Psi, \\
\delta \Psi=-i \gamma^{\mu}\left(\partial_{\mu} \Phi_{a}\right) \epsilon^{a}-i F_{a} \epsilon^{a}, \\
\delta F_{a}=2 \bar{\epsilon}^{a} \gamma^{\mu} \partial_{\mu} \Psi,
\end{gathered}
$$

where $\epsilon^{a}$ 's are a pair of constant four-component spinors satisfying the reality condition:

$$
\epsilon^{1}=-C \epsilon^{2 *}, \quad \epsilon^{2}=C \epsilon^{1 *}, \quad C=\gamma^{1} \gamma^{3} \gamma^{0} .
$$

When a spatial boundary $\partial M$ is inserted at $x^{1}=0$, in the resulting manifold $M$ the set of allowed uniform boundary conditions for the scalars $\Phi_{a}$ 's is again given by (1). But $U_{B}$ in this case is a $2 \times 2$ matrix:

$$
U_{B}=\left(\begin{array}{cc}
U_{B}^{11} & U_{B}^{12} \\
U_{B}^{21} & U_{B}^{22}
\end{array}\right), \quad U_{B}^{\dagger} U_{B}=\mathbb{\natural}_{2 \times 2} .
$$

Therefore, the local boundary conditions on the $\Phi_{a}$ 's are

$$
\left(\Phi_{a}+i \partial_{n} \Phi_{a}\right)_{\partial M}=U_{B}^{a b}\left(\Phi_{b}-i \partial_{n} \Phi_{b}\right)_{\partial M} .
$$

For the choice $U_{B}^{a b}=-\delta^{a b}$ and $U_{B}^{a b}=\delta^{a b}$, we get the Dirichlet and Neumann boundary conditions, respectively. For $U_{B}^{a b} \neq \pm \delta^{a b}$, we get the another type of boundary condition: $\left.\partial_{n} \Phi_{a}\right|_{\partial M}=\left.\kappa^{a b} \Phi_{b}\right|_{\partial M}$, where $\kappa^{a b}=i\left(\left(\square+U_{B}\right)^{-1}\right.$ $\left.\left(\square-U_{B}\right)\right)^{a b}$.

On the spinor $\Psi$, again the boundary conditions (5) and (23) are imposed. But unlike (21) in the $(1+1)$ dimensional massless case, (23) involves the tangential derivatives of $\Psi$ at the boundary. 
The supersymmetry transformation at the boundary must obey

$$
\delta\left(\Phi_{a}+i \partial_{n} \Phi_{a}\right)_{\partial M}=U_{B}^{a b} \delta\left(\Phi_{b}-i \partial_{n} \Phi_{b}\right)_{\partial M} .
$$

Using (51), the above yields

$$
\left[\left(\delta^{a b}-U_{B}^{a b}\right) \bar{\epsilon}^{b} \Psi+i\left(\delta^{a b}+U_{B}^{a b}\right) \bar{\epsilon}^{b} \partial_{n} \Psi\right]_{\partial M}=0 .
$$

Dirichlet boundary condition: If we impose Dirichlet boundary conditions on both the scalars, $U_{B}=-\square_{2 \times 2}$, then (58) and (5) give [similar to the $(1+1)$-dimensional case]

$$
\epsilon_{+}^{a}=-U_{F}^{\dagger} \gamma^{0} \epsilon_{-}^{a} .
$$

Because $\epsilon_{a}$ 's are constant spinors, the above is true not only on the boundary but also in the bulk. Further, using Dirichlet boundary conditions on $\Phi_{a}$ 's and (59), it is easy to check that on the boundary $\partial M$ [similar to the $(1+1)$ dimensional case]

$\delta \Psi_{+}=U_{F} \gamma^{0} \delta \Psi_{+}, \quad \delta\left(H_{D} \Psi\right)_{+}=U_{F} \gamma^{0} \delta\left(H_{D} \Psi\right)_{+}$.

Thus the Dirichlet boundary conditions on both scalars are compatible with supersymmetry transformations. But as the $\epsilon^{a}$ 's are related by (59), the theory is only $\mathcal{N}=1$ supersymmetric.

The closure of the SUSY algebra is governed by

$$
\left[\delta_{\epsilon}, \delta_{\eta}\right]=2 i \bar{\epsilon}_{a} \gamma^{\mu} \eta^{a} \partial_{\mu}+2 i \bar{\epsilon}_{a} \eta^{a} \delta_{Z},
$$

where $\delta_{Z}$ gives the action of the central charge on the fields. The second term in the above vanishes in the massless case and in the massive case it can be absorbed by rescaling the momenta, in a similar fashion as in the $(1+1)$-dimensional case [see (41)].

Neumann and Robin boundary conditions: It is easy to check that if we impose a Neumann- or Robin-type boundary condition on either or both of the scalar fields $\Phi_{a}$ 's, then (5), (23), and (58) cannot be satisfied. Thus such boundary conditions on scalars are not consistent with supersymmetry and SUSY is completely broken.

However, if we insert a boundary $\partial M$ with Dirichlet boundary conditions on scalars, then the theory can still be supersymmetric. The theory with $\mathcal{N}=2$ SUSY breaks to an $\mathcal{N}=1$ supersymmetric theory for every allowed $U_{F}$. For any other choice of boundary conditions, SUSY is completely broken.

\section{EDGE STATES IN $(3+1)$-DIMENSIONS}

In the following we investigate the possibility of the existence of edge states in theories which have residual $\mathcal{N}=1$ SUSY. For simplicity, let us consider the region $x_{3} \leq 0$ as the $(3+1)$-dimensional flat manifold $M$. On the boundary plane $x_{3}=0$, the direction of the outward normal is $\hat{n}=(0,0,1)$. We choose the $\gamma$-matrices in the representation

$\gamma^{\mu}=\left(\begin{array}{ll}0 & \sigma^{\mu} \\ \bar{\sigma}^{\mu} & 0\end{array}\right), \quad \sigma^{\mu}=\left(1, \sigma^{i}\right), \quad \bar{\sigma}^{\mu}=\left(1,-\sigma^{i}\right)$.

In this case $U_{F}$ satisfies

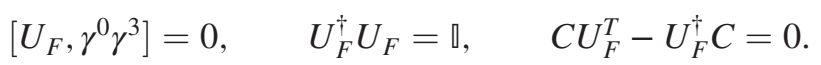

The last condition in the above is imposed by (54) and (59). Therefore, the most general $U_{F}$ in this case is given by (a detailed derivation is given in Appendix A)

$U_{F}=\left(\begin{array}{cccc}v_{1} & 0 & 0 & v_{2} \\ 0 & u_{1}^{*} & -u_{2}^{*} & 0 \\ 0 & u_{2} & u_{1} & 0 \\ -v_{2}^{*} & 0 & 0 & v_{1}^{*}\end{array}\right), \quad u_{1}, u_{2}, v_{1}, v_{2} \in \mathbb{C}$

with $\left|u_{1}\right|^{2}+\left|u_{2}\right|^{2}=1$ and $\left|v_{1}\right|^{2}+\left|v_{2}\right|^{2}=1$.

(i) Massless case: In the massless case, if $u_{1}=0$, $\operatorname{Re}\left(u_{2}\right) \neq 0$, and $\operatorname{Im}\left(u_{2}\right) \neq 0$ are chosen in (64), there exist two zero-energy edge localized states for arbitrary $b>0$ :

$$
\Psi_{e_{1}}^{0}=A_{k}\left(\begin{array}{c}
1 \\
-u_{2 *} \\
0 \\
0
\end{array}\right) e^{b x^{3}+i k_{1} x^{1}+i k_{2} x^{2}}
$$

with $k_{1}=b \operatorname{Im}\left(u_{2}\right), k_{2}=b \operatorname{Re}\left(u_{2}\right)$, and

$$
\Psi_{e_{2}}^{0}=D_{k}\left(\begin{array}{c}
0 \\
0 \\
u_{2} \\
1
\end{array}\right) e^{b x^{3}+i k_{1} x^{1}+i k_{2} x^{2}}
$$

with $k_{1}=-b \operatorname{Im}\left(u_{2}\right), k_{2}=-b \operatorname{Re}\left(u_{2}\right) . A_{k}$ and $D_{k}$ are normalization constants. As $\Psi_{e_{1}}^{0 \dagger} \Psi_{e_{2}}^{0}=0$, these two modes are linearly independent. For sufficiently large $b$, these modes are localized near the edge and are exponentially damped in the bulk.

For this choice of $u_{1}$ and $u_{2}$, there does not exist any other normalizable zero-energy edge state.

(ii) Massive case: If we choose $\operatorname{Re}\left(u_{1}\right)=0, \operatorname{Im}\left(u_{1}\right) \neq 0$, $\operatorname{Re}\left(u_{2}\right) \neq 0$, and $\operatorname{Im}\left(u_{2}\right) \neq 0$ in (64), there exist either of the following two zero-energy states: 
(a) For $\operatorname{Im}\left(u_{1}\right)<0$,

$$
\Psi_{e_{1}}^{m}=A_{k}\left(\begin{array}{c}
1 \\
-u_{2}^{*} \\
u_{1} \\
0
\end{array}\right) e^{b x^{3}+i k_{1} x^{1}+i k_{2} x^{2}},
$$

with $b=-\frac{m}{\operatorname{Im}\left(u_{1}\right)}, k_{1}=b \operatorname{Im}\left(u_{2}\right)$, and $k_{2}=b \operatorname{Re}\left(u_{2}\right)$. ( $A_{k}$ is the normalization constant.)

(b) For $\operatorname{Im}\left(u_{1}\right)>0$,

$$
\Psi_{e_{2}}^{m}=D_{k}\left(\begin{array}{c}
0 \\
u_{1}^{*} \\
u_{2} \\
1
\end{array}\right) e^{b x^{3}+i k_{1} x^{1}+i k_{2} x^{2}},
$$

with $b=\frac{m}{\operatorname{Im}\left(u_{1}\right)}, k_{1}=-b \operatorname{Im}\left(u_{2}\right)$, and $k_{2}=-b \operatorname{Re}\left(u_{2}\right) .\left(D_{k}\right.$ is the normalization constant.) If $m$ is very large and/or $\left|\operatorname{Im}\left(u_{1}\right)\right|$ is very small, these states are exponentially damped in the bulk and are localized near the edge.

For these choices of $u_{1}$ and $u_{2}$, there does not exist any other normalizable edge state.

For a scalar field obeying Dirichlet boundary conditions, there are no zero-energy modes of the Laplacian (for details see Appendix B). On the other hand, it is possible to choose boundary conditions for the fermion such that there exist fermionic zero modes. In such a situation, the ground state is made up of a fermion but no boson. This however does not break supersymmetry, precisely because it is a zeroenergy state.

If such fermionic edge states exist, it should be possible to experimentally detect them in condensed matter systems, especially in the supersymmetric phase of superconductors.

\section{NONLOCAL BOUNDARY CONDITIONS}

Although the main goal of this work is to analyze the possibility of existence of edge states in supersymmetric theories associated to topological superconductors, since the problem of SUSY breaking by boundary conditions has been addressed in other contexts we shall extend our analysis to more general boundary conditions. In the case of topological superconductors all relevant physical conditions are local and they were analyzed in the previous sections. However, it is well known that in superstring theory SUSY is preserved by periodic boundary conditions. These kinds of boundary conditions are highly nonlocal and the natural question one can raise is, Are there other nonlocal boundary conditions which preserve SUSY totally or partially? We shall focus now on this problem.

\section{A. Periodic boundary conditions on both scalars and fermions}

Consider $(1+1)$-dimensional $\mathcal{N}=2$ supersymmetric theory with action (6) and SUSY transformations (9).
We introduce spatial boundaries at $x_{1}=0$ and $x_{1}=a$. In the manifold

$$
M=\left\{\left(x^{0}, x^{1}\right): 0 \leq x^{1} \leq a\right\},
$$

it is easy to see that boundary conditions

$$
\begin{array}{ll}
\left.\Phi\right|_{x_{1}=0}=\left.\Phi\right|_{x_{1}=a}, & \left.\partial_{1} \Phi\right|_{x_{1}=0}=\left.\partial_{1} \Phi\right|_{x_{1}=a}, \\
\left.\Psi\right|_{x_{1}=0}=\left.\Psi\right|_{x_{1}=a} &
\end{array}
$$

give a self-adjoint domain of $H_{s}$ and $H_{D}$.

With these boundary conditions, it is straightforward to show that the SUSY transformations (9) satisfy

$$
\left.\delta \Phi\right|_{x_{1}=0}=\left.\delta \Phi\right|_{x_{1}=a},\left.\quad \delta \Psi\right|_{x_{1}=0}=\left.\delta \Psi\right|_{x_{1}=a}
$$

without any condition $\epsilon$.

Hence, these boundary conditions are consistent with SUSY transformations and preserve $\mathcal{N}=2$ SUSY. They describe a closed superstring with Ramond-Ramond (RR) boundary conditions. It is quite easy to extend the above to higher dimensions and the result holds true there as well.

\section{B. Periodic boundary conditions on scalars and antiperiodic boundary conditions on fermions}

However, from a physical viewpoint it is more natural to consider periodic boundary conditions on the scalars and antiperiodic ones for fermions. In field theories at finite temperature these are the natural boundary conditions, because positivity preserving requires antiperiodic boundary conditions for fermions in the temporal direction, and by Euclidean symmetry it is also convenient to have such conditions also in space dimensions [13].

Again, we consider a $(1+1)$-dimensional $\mathcal{N}=2$ supersymmetric theory with action (6) and SUSY transformations (9). On introduction of spatial boundaries at $x_{1}=0$ and $x_{1}=a$, it is easy to see that on the manifold

$$
M=\left\{\left(x^{0}, x^{1}\right): 0 \leq x^{1} \leq a\right\},
$$

the boundary conditions

$$
\begin{aligned}
& \left.\Phi\right|_{x_{1}=0}=\left.\Phi\right|_{x_{1}=a},\left.\quad \partial_{1} \Phi\right|_{x_{1}=0}=\left.\partial_{1} \Phi\right|_{x_{1}=a}, \\
& \left.\Psi\right|_{x_{1}=0}=-\left.\Psi\right|_{x_{1}=a}
\end{aligned}
$$

give a self-adjoint domain of $H_{S}$ and $H_{D}$.

On demanding that the SUSY transformations are consistent with the boundary conditions, we get

$$
\left.\delta \Phi\right|_{x_{1}=0}=\left.\delta \Phi\right|_{x_{1}=a} .
$$

This leads to

$$
\epsilon\left[\left.\Psi\right|_{x_{1}=0}-\left.\Psi\right|_{x_{1}=a}\right]=0 .
$$

This is inconsistent with the fermionic boundary condition $\left.\Psi\right|_{x_{1}=0}=-\left.\Psi\right|_{x_{1}=a}$. 
Therefore, when such boundary conditions are imposed on the fields, SUSY is completely broken. It is easy to see that the same result is valid in any spacetime dimension.

\section{Dirichlet boundary condition on scalar and (anti) periodic boundary condition on fermion}

Again consider the action (6) and SUSY transformations (9) on the manifold

$$
M=\left\{\left(x^{0}, x^{1}\right): 0 \leq x^{1} \leq a\right\} .
$$

If we impose

$$
\left.\Phi\right|_{x_{1}=0}=0=\left.\Phi\right|_{x_{1}=a},\left.\quad \Psi\right|_{x_{1}=0}= \pm\left.\Psi\right|_{x_{1}=a},
$$

then the supersymmetry transformations (9) lead to

$$
\epsilon\left[\left.\left.\partial_{1} \Phi\right|_{x_{1}=0} \mp \partial_{1} \Phi\right|_{x_{1}=a}\right]=0 .
$$

The above can be satisfied only if we demand

$$
\left.\partial_{1} \Phi\right|_{x_{1}=0}= \pm\left.\partial_{1} \Phi\right|_{x_{1}=a} .
$$

Therefore, the requirement to preserve SUSY is that the domain of $H_{s}$ must be

$$
\begin{aligned}
\mathcal{D}_{H_{s}} & =\left\{\Phi:\left.\Phi\right|_{x_{1}=0}=0=\left.\Phi\right|_{x_{1}=a},\right. \\
\left.\partial_{1} \Phi\right|_{x_{1}=0} & \left.= \pm\left.\partial_{1} \Phi\right|_{x_{1}=a}\right\} .
\end{aligned}
$$

However, this is not a self-adjoint domain of $H_{s}$. Hence, we cannot demand (79). Thus the boundary conditions (77) are not consistent with (9) and imposing such boundary conditions will break SUSY completely.

\section{Neumann boundary condition on scalar and (anti) periodic boundary condition on fermion}

Again consider the action (6) and SUSY transformations (9) on the manifold

$$
M=\left\{\left(x^{0}, x^{1}\right): 0 \leq x^{1} \leq a\right\} .
$$

If we impose

$$
\left.\partial_{1} \Phi\right|_{x_{1}=0}=0=\left.\partial_{1} \Phi\right|_{x_{1}=a},\left.\quad \Psi\right|_{x_{1}=0}= \pm\left.\Psi\right|_{x_{1}=a},
$$

then the supersymmetry transformations (9) lead to

$\partial_{0}\left[\gamma^{0} \epsilon\left(\left.\left.\Phi\right|_{x_{1}=0} \mp \Phi\right|_{x_{1}=a}\right)\right]+m \epsilon\left[\left.\left.\Phi\right|_{x_{1}=0} \mp \Phi\right|_{x_{1}=a}\right]=0$.

The above can be satisfied only if we demand

$$
\left.\Phi\right|_{x_{1}=0}= \pm\left.\Phi\right|_{x_{1}=a} .
$$

Therefore, the requirement to preserve SUSY is that the domain of $H_{s}$ must be

$$
\begin{aligned}
\mathcal{D}_{H_{s}} & =\left\{\Phi:\left.\partial_{1} \Phi\right|_{x_{1}=0}=0=\left.\partial_{1} \Phi\right|_{x_{1}=a},\right. \\
\left.\Phi\right|_{x_{1}=0} & \left.= \pm\left.\Phi\right|_{x_{1}=a}\right\} .
\end{aligned}
$$

Again, this is not a self-adjoint domain of $H_{s}$. Hence, we cannot demand (84). Thus the boundary conditions (82) are not consistent with (9) and imposing such boundary conditions will break SUSY completely.

In a similar fashion, one can show that imposing a Robin boundary condition on the scalar and (anti)periodic boundary condition on the fermion also breaks SUSY completely. These results are also valid in any spacetime dimension.

\section{E. Periodic boundary conditions on scalar and local boundary conditions on fermion}

Consider the action (6) and SUSY transformations (9) on the manifold

$$
M=\left\{\left(x^{0}, x^{1}\right): 0 \leq x^{1} \leq a\right\} .
$$

The boundary conditions

$$
\begin{aligned}
\left.\Phi\right|_{x_{1}=0} & =\left.\Phi\right|_{x_{1}=a},\left.\quad \partial_{1} \Phi\right|_{x_{1}=0}=\left.\partial_{1} \Phi\right|_{x_{1}=a}, \\
\left.\Psi_{+}\right|_{x_{1}=0} & =\left.U_{F}\left(x_{1}=0\right) \Psi_{+}\right|_{x_{1}=0}, \\
\left.\Psi_{+}\right|_{x_{1}=a} & =\left.U_{F}\left(x_{1}=a\right) \Psi_{+}\right|_{x_{1}=a}
\end{aligned}
$$

give a self-adjoint domain of $H_{s}$ and $H_{D}$.

For the above boundary conditions to be consistent with SUSY transformations, the following must be satisfied:

$$
\left.\delta \Phi\right|_{x_{1}=0}=\left.\delta \Phi\right|_{x_{1}=a} .
$$

This yields

$$
\bar{\epsilon}\left[\left.\Psi\right|_{x_{1}=0}-\left.\Psi\right|_{x_{1}=a}\right]=0,
$$

which can only be satisfied if we further impose

$$
\left.\Psi\right|_{x_{1}=0}=\left.\Psi\right|_{x_{1}=a} .
$$

However, imposing this condition on $\Psi$ shrinks the domain of $H_{D}$ and in that shrunk domain, $H_{D}$ is not self-adjoint. Hence, (90) cannot be imposed. Thus the boundary conditions (87) cannot be made consistent with the supersymmetry transformation (9). As a result, imposing (87) will break SUSY completely.

One can easily show that these results are also valid in any spacetime dimension.

\section{F. Other nonlocal boundary conditions}

Finally, we discuss some boundary conditions which are similar to Ramond and Neveu-Schwarz boundary conditions that are well known in the context of string theory. 
Consider $(1+1)$-dimensional $\mathcal{N}=2$ supersymmetric theory with action (6) and SUSY transformations (9) in the manifold

$$
M=\left\{\left(x^{0}, x^{1}\right): 0 \leq x^{1} \leq a\right\} .
$$

The Dirac operator is

$$
H_{D}=i \gamma^{0} \gamma^{1} \partial_{1}-m \gamma^{0}
$$

We choose the representation of the $\gamma$-matrices

$$
\gamma^{0}=\sigma^{2}, \quad \gamma^{1}=i \sigma^{1},
$$

where $\sigma$ 's are the Pauli matrices.

For any $\Psi \in \mathcal{D}_{H_{D}}$ and $\xi \in \mathcal{D}_{H_{D}}$, the self-adjointness demands that the conditions

$$
\left.\xi^{\dagger} \sigma^{3} \Psi\right|_{x_{1}=a}-\left.\xi^{\dagger} \sigma^{3} \Psi\right|_{x_{1}=a}=0
$$

are satisfied with the same condition on $\Psi$ and $\xi$.

$\Psi$ and $\xi$ are two-component Dirac spinors:

$$
\Psi=\left(\begin{array}{l}
\Psi_{1} \\
\Psi_{2}
\end{array}\right), \quad \xi=\left(\begin{array}{l}
\xi_{1} \\
\xi_{2}
\end{array}\right) .
$$

In terms of the components, the condition (94) can be written as

$$
\left[\xi_{1}^{\dagger} \Psi_{1}-\xi_{2}^{\dagger} \Psi_{2}\right]_{x_{1}=a}-\left[\xi_{1}^{\dagger} \Psi_{1}-\xi_{2}^{\dagger} \Psi_{2}\right]_{x_{1}=0}=0 .
$$

We discuss two types of boundary conditions which can satisfy the above.

\section{Pseudoperiodic boundary conditions}

It can be easily seen that (96) can be satisfied by the boundary condition

$$
\left.\begin{array}{l}
\left.\Psi_{1}\right|_{x_{1}=a}=\left.e^{i \theta_{1}} \Psi_{1}\right|_{x_{1}=0}, \\
\left.\Psi_{2}\right|_{x_{1}=a}=\left.e^{i \theta_{2}} \Psi_{2}\right|_{x_{1}=0},
\end{array}\right\} \quad \theta_{1}, \theta_{2} \in \mathbb{R}
$$

Hence,

$$
\begin{aligned}
\mathcal{D}_{H_{D}} & =\left\{\Psi:\left.\Psi_{1}\right|_{x_{1}=a}=\left.e^{i \theta_{1}} \Psi_{1}\right|_{x_{1}=0},\right. \\
\left.\Psi_{2}\right|_{x_{1}=a} & \left.=\left.e^{i \theta_{2}} \Psi_{2}\right|_{x_{1}=0}\right\}
\end{aligned}
$$

is a self-adjoint domain of $H_{D}$.

The supersymmetry transformation (9) can only be made compatible with the boundary condition (97) if we impose

$$
\theta_{1}=\theta_{2}=\theta,\left.\Phi\right|_{x_{1}=a}=\left.e^{i \theta} \Phi\right|_{x_{1}=0},\left.\partial_{1} \Phi\right|_{x_{1}=a}=\left.e^{i \theta} \partial_{1} \Phi\right|_{x_{1}=0} .
$$

It is easy to see that
$\mathcal{D}_{H_{s}}=\left\{\Phi:\left.\Phi\right|_{x_{1}=a}=\left.e^{i \theta} \Phi\right|_{x_{1}=0},\left.\quad \partial_{1} \Phi\right|_{x_{1}=a}=\left.e^{i \theta} \partial_{1} \Phi\right|_{x_{1}=0}\right\}$

is a self-adjoint domain of $H_{s}$.

Hence, these boundary conditions are consistent with SUSY transformations and preserve $\mathcal{N}=2$ SUSY. The cases considered in Sec. VIA are special cases of these conditions with $\theta=n \pi$ where $n \in \mathbb{Z}$.

\section{CONCLUSIONS AND DISCUSSIONS}

We have shown that SUSY can be broken when spatial boundaries are introduced in an $\mathcal{N}=2$ supersymmetric theory. Only very few boundary conditions partially preserve SUSY. For most boundary conditions, SUSY is completely broken.

The results for $(1+1)$-dimensional systems are summarized in the following Table I.

Similar results were obtained in two-dimensional kink backgrounds with space boundaries as infrared cutoffs [14]. The breaking of SUSY by effect of branes and/or domain walls is well known and can be related to our results (see, e.g., [15] and references therein). However, the most interesting result is that edge states can appear even if SUSY is not completely broken.

As we have shown, it is possible to extend our analysis to any spacetime dimension. Though we have considered only flat boundaries for the simplicity of our analysis, it is not difficult to see that the results will be true in general, for any curved boundary. Also, the above analysis is valid not only for $\mathcal{N}=2$, but also for any $\mathcal{N}=2 q$ (even) supersymmetric theory. In our analysis, we considered free theories. However, one might consider interactions as well and in that case, it is not difficult to convince oneself that the results should be consistent with [16]. We expect that edge states in these interacting theories (more realistic in the context of, say, a superconductor) exist in a similar fashion.

TABLE I. Boundary conditions and SUSY in $1+1$ dimensions.

\begin{tabular}{lccccc}
\hline \hline Boson & Fermion & $\mathrm{m}$ & $\mathcal{N}=2$ & $\mathcal{N}=1$ & $\begin{array}{c}\text { Edge } \\
\text { states }\end{array}$ \\
\hline Dirichlet & $U_{F}(\theta, \tilde{\theta})$ & 0 & No & Yes & No \\
& & $\neq 0$ & & & Yes \\
Neumann & $U_{F}(\theta, \tilde{\theta})$ & 0 & No & Yes & No \\
& $U_{F}= \pm I$ & $\neq 0$ & & & Yes \\
Dirichlet & Periodic & Any & No & No & No \\
Neumann & Periodic & Any & No & No & No \\
Periodic & $U_{F}(\theta, \tilde{\theta})$ & Any & No & No & No \\
& $\times U_{F}(\theta, \tilde{\theta})$ & & & & \\
& Antiperiodic & & No & No & \\
& Periodic & & Yes & Yes & \\
Antiperiodic & Antiperiodic & Any & Yes & Yes & No \\
Pseudoperiodic & Pseudoperiodic & Any & Yes & Yes & No \\
\hline \hline
\end{tabular}


Nonetheless, the details of the properties of these states need to be studied.

The presence of the edge localized fermions as ground states of these supersymmetric theories is important in the context of systems like topological superconductors. For example, these fermions localized on the boundary will contribute to the Meissner effect of the superconductor and thus experimental verification of these fermions localized in the boundary is possible.

\section{ACKNOWLEDGMENTS}

We would like to thank Romesh Kaul and Subroto Mukerjee for illuminating discussions and suggestions. A. P. B. thanks the Centre for High Energy Physics, Indian Institute Science, Bangalore, for hospitality during the course of this work. He also thanks Andrés Reyes at the Universidad de los Andes for hosting him as the Sanford Professor and for warm hospitality when this work was being completed. The work of M. A. has been partially supported by Spanish DGIID-DGA Grant No. 2014-E24/2 and Spanish MICINN Grants No. FPA2012-35453 and No. CPAN-CSD2007-00042.

\section{APPENDIX A: THE MOST GENERAL $\boldsymbol{U}_{\boldsymbol{F}}$}

In the $(3+1)$-dimensional manifold $M=\left\{x_{3} \leq 0\right\}, U_{F}$ is of the form

$$
U_{F}=\left(\begin{array}{ll}
U_{1} & U_{2} \\
U_{3} & U_{4}
\end{array}\right),
$$

where $U_{1}, U_{2}, U_{3}$, and $U_{4}$ are $2 \times 2$ matrices. Also $U_{F}$ satisfies

$\left[U_{F}, \gamma^{0} \gamma^{3}\right]=0, \quad C U_{F}^{T}-U_{F}^{\dagger} C=0, \quad U_{F}^{\dagger} U_{F}=\emptyset$.

With the choice of $\gamma$-matrices as in (62), the condition $\left[U_{F}, \gamma^{0} \gamma^{3}\right]=0$ yields

$$
\left(\begin{array}{cc}
-\left[U_{1}, \sigma^{3}\right] & \left\{U_{2}, \sigma^{3}\right\} \\
-\left\{U_{3}, \sigma^{3}\right\} & {\left[U_{4}, \sigma^{3}\right]}
\end{array}\right)=0 .
$$

Hence,

$$
\begin{aligned}
& {\left[U_{1}, \sigma^{3}\right]=0, \quad\left\{U_{2}, \sigma^{3}\right\}=0, \quad\left\{U_{3}, \sigma^{3}\right\}=0,} \\
& {\left[U_{4}, \sigma^{3}\right]=0 .}
\end{aligned}
$$

Therefore, the most general $U_{1}$ and $U_{4}$ are

$$
U_{1}=\left(\begin{array}{cc}
v_{1} & 0 \\
0 & v_{4}
\end{array}\right), \quad U_{4}=\left(\begin{array}{cc}
u_{1} & 0 \\
0 & u_{4}
\end{array}\right),
$$

where $u_{1}, v_{1}, u_{4}, v_{4} \in \mathbb{C}$ and the most general $U_{2}$ and $U_{3}$ are

$$
U_{2}=\left(\begin{array}{cc}
0 & v_{2} \\
v_{3} & 0
\end{array}\right), \quad U_{3}=\left(\begin{array}{cc}
0 & u_{2} \\
u_{3} & 0
\end{array}\right),
$$

where $u_{2}, v_{2}, u_{3}, v_{3} \in \mathbb{C}$.

From (A5), it is easy to see that

$U_{1}^{\dagger}=U_{1}^{*}, \quad U_{1}^{T}=U_{1}, \quad U_{4}^{\dagger}=U_{4}^{*}, \quad U_{4}^{T}=U_{4}$.

Hence, the condition $C U_{F}^{T}-U_{F}^{\dagger} C=0$ leads to

$$
\sigma^{2} U_{2}^{T}-U_{3}^{\dagger} \sigma^{2}=0, \quad \sigma^{2} U_{4}-U_{1}^{*} \sigma^{2}=0 .
$$

These yield

$$
v_{4}=u_{1}^{*}, \quad u_{4}=v_{1}^{*}, \quad v_{3}=-u_{2}^{*}, \quad u_{3}=-v_{2}^{*} .
$$

Therefore,

$U_{F}=\left(\begin{array}{cccc}v_{1} & 0 & 0 & v_{2} \\ 0 & u_{1}^{*} & -u_{2}^{*} & 0 \\ 0 & u_{2} & u_{1} & 0 \\ -v_{2}^{*} & 0 & 0 & v_{1}^{*}\end{array}\right), \quad u_{1}, u_{2}, v_{1}, v_{2} \in \mathbb{C}$.

The unitarity condition $U_{F}^{\dagger} U_{F}=\rrbracket$ gives

$$
\left|v_{1}\right|^{2}+\left|v_{2}\right|^{2}=1, \quad\left|u_{1}\right|^{2}+\left|u_{2}\right|^{2}=1 .
$$

\section{APPENDIX B: ZERO MODES OF THE SCALAR FIELD}

In the $(3+1)$-dimensional manifold $M=\left\{x_{3} \leq 0\right\}$, the zero modes of the scalar field are

$$
\begin{gathered}
\left(-\nabla^{2}+m^{2}\right) \Phi(x)=0, \\
\Phi(x)=\left(A_{k} e^{b x_{3}}+B_{k} e^{-b x_{3}}\right) e^{i\left(k_{1} x_{1}+k_{2} x_{2}\right)},
\end{gathered}
$$

with

$$
b^{2}=k_{1}^{2}+k_{2}^{2} .
$$

As $x_{3} \rightarrow-\infty$, $\Phi$ must go to zero. Hence, $B_{k}=0$ and

$$
\Phi(x)=A_{k} e^{b x_{3}+i\left(k_{1} x_{1}+k_{2} x_{2}\right)} .
$$

Imposing the Dirichlet boundary condition at $x_{3}=0$,

$$
\left.\Phi(x)\right|_{\partial M}=0,
$$

yields

$$
A_{k}=0 .
$$

Hence, there are no zero-energy scalar modes. 
[1] T. Grover, D. N. Sheng, and A. Vishwanath, Emergent space-time supersymmetry at the boundary of a topological phase, Science 344, 280 (2014).

[2] X.-L. Qi and S.-C. Zhang, Topological insulators and superconductors, Rev. Mod. Phys. 83, 1057 (2011).

[3] D. V. Belyaev and P. van Nieuwenhuizen, Rigid supersymmetry with boundaries, J. High Energy Phys. 04 (2008) 008.

[4] P. van Nieuwenhuizen and D. V. Vassilevich, Consistent boundary conditions for supergravity, Classical Quantum Gravity 22, 5029 (2005).

[5] M. Asorey, D. Garcia-Alvarez, and J. M. Munoz-Castaneda, Casimir effect and global theory of boundary conditions, J. Phys. A 39, 6127 (2006).

[6] M. Asorey and J. M. Munoz-Castaneda, Vacuum boundary effects, J. Phys. A 41, 304004 (2008).

[7] M. Asorey and J. M. Munoz-Castaneda, Vacuum boundary effects, Int. J. Theor. Phys. 50, 2211 (2011).

[8] M. Asorey, D. Garcia-Alvarez, and J. M. Munoz-Castaneda, Boundary effects in bosonic and fermionic field theories, Int. J. Geom. Methods Mod. Phys. 12, 1560004 (2015).
[9] M. Asorey, A. Ibort, and G. Marmo, Global theory of quantum boundary conditions and topology change, Int. J. Mod. Phys. A20, 1001 (2005).

[10] M. Asorey, A. P. Balachandran, and J. M. Perez-Pardo, Edge states: Topological insulators, superconductors and QCD chiral bags, J. High Energy Phys. 12 (2013) 073.

[11] K. Hori, Trieste lectures on mirror symmetry, Spring School on Superstrings and Related Matters (2012).

[12] M. Sohnius, Introducing supersymmetry, Phys. Rep. 128, 39 (1985).

[13] E. Witten, Anti-de Sitter space, thermal phase transition, and confinement in gauge theories, Adv. Theor. Math. Phys. 2, 505 (1998).

[14] M. A. Shifman, A. I. Vainshtein, and M. B. Voloshin, Anomaly and quantum corrections to solitons in twodimensional theories with minimal supersymmetry, Phys. Rev. D 59, 045016 (1999).

[15] J. L. Lehners, P. Smyth, and K. S. Stelle, Kaluza-Klein induced supersymmetry breaking for braneworlds in type IIB supergravity, Nucl. Phys. B790, 89 (2008).

[16] D. Gaiotto and E. Witten, Supersymmetric boundary conditions in $N=4$ super Yang-Mills theory, J. Stat. Phys. 135, 789 (2009). 